\title{
Wikipedia und die Transformation der Wissensaushandlung
}

\author{
Johannes Gemkow
}

Keywords: Wissen, Medienkompetenz, Wikipedia, Macht

\section{Abstract}

Die Wikipedia gilt als Paradespiel für kollektive Wissensherstellung. Die Generierung und Prozessierung von Wissen basiert dort aufverschiedenen Handlungsmöglichkeiten und -bedingungen. Im Zusammenspiel mit letzteren treten verschiedene Akteur*innen auf, die durch unterschiedliche Strukturen legitimiert, Einfluss auf das von der Wikipedia repräsentierte Wissen nehmen. Zwischen den Akteur*innen und Strukturen, welche die Handlungen gestalten, entsteht ein Kräfteverhältnis. Dieses kann im Anschluss an Foucault als produktive Macht verstanden werden. Der Beitrag zielt auf die Skizzierung selbiger auf der Wikipedia ab und stellt ihr das medienpädagogische Konzept der Medienkompetenz gegenüber.

Johannes Gemkow: Wikipedia und die Transformation der Wissensaushandlung. In: Nils S. Borchers, Selma Güney, Uwe Krüger und Kerem Schamberger (Hrsg.): Transformation der Medien - Medien der Transformation. Verhandlungen des Netzwerks Kritische Kommunikationswissenschaft. Frankfurt am Main: Westend 2021. DOI: https://doi.org/10.53291/TLAJ4696.

Dr. Johannes Gemkow I Universität Leipzig I johannes.gemkow@uni-leipzig.de 
Dieser Beitrag zielt auf die Skizzierung der produktiven Macht auf der Wikipedia ab. Dabei wird er nicht auf einer soziologischen Deskription ebenjener Macht verweilen, sondern mit dem Konzept der Medienkompetenz ein pädagogisches Korrektiv diskutieren. Dafür ist die produktive Macht von doppelter Bedeutung: Erstens werden über sie Möglichkeiten der Partizipation ausgehandelt, und zweitens hat das von Wikipedia repräsentierte Wissen einen erheblichen Stellenwert für die Praxis des informellen Lernens. Das klassische Konzept der Medienkompetenz kann diese beiden Aspekte inhaltlich vereinen, müsste sich dafür aber von seinem - im Folgenden noch skizzierten - idealistischen (vgl. Gapski 2001) und subjektspezifischen (vgl. Baacke 1998) Impetus lösen, um hin zu einer gegenständlichen Verflechtung von Akteur*innen, Strukturen und Plattform zu gelangen.

Der Beitrag wird über den in Abschnitt 3 skizzierten Machtbegriff hinausgehend bei der Unterscheidung von Handlungsmöglichkeiten und -bedingungen auf das sozialwissenschaftliche Dispositivverständnis zurückgreifen (vgl. Bührmann und Schneider 2012; Gemkow 2017). Für das Konzept der Medienkompetenz schließt er an die in der Medienpädagogik aktuelle Debatte um eine Neuakzentuierung des Konzeptes an (vgl. Zorn 2011; Schiefner-Rohs 2012; Walden 2017; Bettinger und Aßmann 2017).

Durch seine Beschreibung, wie in der Wikipedia Wissen hervorgebracht wird, leistet der Artikel einen Beitrag zur Transformation der Wissensaushandlung. Diese bezieht sich dabei auf die historisch und soziotechnisch spezifische Konstellation, mit der sich über die Plattform eine Generierungs- und Strukturierungsweise von Wissen etabliert hat.

\section{Die Wikipedia}

Die Online-Enzyklopädie Wikipedia enthält sprachübergreifend mehr als 36 Millionen Artikel, wobei die englische Version mit knapp über 5 Millionen am umfangreichsten ist. Auf der deutschen Wikipedia gibt es zum Vergleich etwa die Hälfte. Demgegenüber stellt die aktuelle Auflage des Brockhaus - sowohl Online als auch Print - rund 300000 Artikel bereit (vgl. Wikipedia 2020a). Im Januar 2020 wurde die deutsche Version täglich ungefähr 36 Millionen Mal aufgerufen. Die englische Wikipedia kam im selben Zeitraum auf etwa 265 Millionen Aufrufe und 
alle Sprachversionen zusammengenommen auf rund 537 Millionen (vgl. Wikipedia 2020d). Damit stellt sie allein mithin das größte Angebot an Wissensrepräsentationen im World Wide Web dar.

Ebenfalls bietet die Wikipedia eine niedrige Zugangsschwelle. Sofern die Nutzer*innen über Computer, Internetverbindung und Browser verfügen, muss keine zusätzliche Software angeschafft geschweige denn bezahlt werden. Wikis sind zudem in der Regel ohne tiefgreifende technische Kenntnisse nutzbar (vgl. Arnold et al. 2018, 228).

Die zugrundeliegende Software ermöglicht, dass alle Themenbereiche unter einem gemeinsamen technischen Konzept stehen, welches bestimmte Handlungen ermöglicht und andere ausschließt. Einzelne Weblogs, E-Learning-Systeme oder Open Educational Resources müssen beispielsweise nicht zwangsläufig demselben technischen Konzept unterliegen. Die Wiki-Software verfügt dahingehend über einen relativ homogenen Pool an Generierungs- und Strukturierungsweisen von Wissen. Als bekannteste Vertreterin bietet die Wikipedia selbst ebenfalls keine thematische Spezifizierung. Ihre Funktionsweise erlaubt das Verarbeiten, also Prozessieren von Wissensrepräsentationen, durch ein kollaborativ arbeitendes Kollektiv (vgl. Cunningham 2002).

Durch die nutzergesteuerte Bearbeitung und Veröffentlichung verwirklicht die Wiki-Software Aspekte wie kontinuierlichen Prozess, Heterarchie, Dezentralisierung oder Interaktion, die häufig mit der Digitalisierung verbunden werden (vgl. Pscheida 2010, 443). Das Konzept der Wikis, und die Wikipedia als deren Hauptvertreterin, ist damit ein Paradebeispiel für den Gegenstand des digitalisierten Wissens. Ähnlich wie das Manuskript während der Literalisierung oder das Buch während der Typographisierung von Wissen wurde mit ihnen eine Technik geschaffen, welche die historisch spezifische Generierung und Strukturierung von Wissen repräsentiert.

Die Wikipedia gilt folglich auch als Paradespiel für kollektive Wissensherstellung. Einerseits kommt ihr eine euphorische Zuschreibung als Verwirklichung des Traumes eines kollektiven menschlichen Gedächtnisses zu, andererseits werden aber auch negative Perspektiven wie der Verlust individueller Gedächtnisleistungen und fehlende Qualitätssicherung mit der Wikipedia verknüpft. 


\section{Die produktive Macht (auf) der Wikipedia}

Das Konzept der produktiven Macht zeichnet sich durch unterschiedliche Aspekte aus. Der erste ist, dass sie niemandem gehört: »[Ü]berall, wo es Macht gibt, wird Macht ausgeübt. Niemand ist im Grunde Inhaber der Macht; und dennoch wird sie stets in eine bestimmte Richtung ausgeübt.« (Foucault 2002, 389-390) In diesem Sinne funktioniert sie »über eine netzförmige Organisation« (Foucault 1978, 82). Dieses Verständnis von Macht ist auch auf die Wissensgenerierung und -strukturierung der Wikipedia anwendbar, da auf keine zentrale, autoritäre Institution verwiesen werden kann. Vielmehr lässt sich ein Zusammenspiel verschiedener Akteur*innen und Wahrheitsansprüche beobachten.

Der zweite wichtige Aspekt der produktiven Macht ist in ihrer Wirkung zu sehen. Weit mehr als ein Netz von Kräfteverhältnissen ist »die Macht produktiv; und sie produziert Wirkliches. Sie produziert Gegenstandsbereiche und Wahrheitsrituale: das Individuum und seine Erkenntnis sind Ergebnisse dieser Produktion« (Foucault 1994, 250). Die produktive Macht konstituiert also selbst das Feld, in dem sie agiert, und etabliert verschiedene Handlungspraktiken. In diesem Sinne stützt sie sich auf »dauerhafte Strukturen « und besteht aus einem »Ensemble von Handlungen « (Foucault 2005, 256). Macht ist also nicht unterwerfend, sondern positioniert Handlungen, Akteur*innen und deren jeweiligen Gegenstandsbereich.

Im Falle der Wikipedia bildet dieser Gegenstandbereich die digitalen Wissensrepräsentationen. Diese sind damit das Objekt der Analyse, jedoch nicht das Wissen im Foucault'schen Sinn selbst, welches er als »einen Prozess, der das Subjekt einer Veränderung unterwirft« (Foucault 1996, 52) definiert. Dieses Verständnis ermöglicht es, Wissen entweder als subjektiven Lernprozess zu deuten (auch im Sinne von Widerstand gegenüber der Macht) oder als Subjektivierung - also eine etablierte Vorstellung der Art und Klassifikation von Subjekten. Die digitalen Wissensrepräsentationen hingegen sind nicht mit diesem Wissensverständnis gleichzusetzen, sondern bilden den austauschbaren Objektbereich, der durch die produktive Macht der Wikipedia hervorgebracht wird.

Hier stellt sich die Frage, wie diese produktive Macht zu analysieren ist. Der Ausgangspunkt setzt mit Foucault nicht beim Objekt (hier: digitale Wissensrepräsentationen) an, sondern bei den Umständen, die dieses Objekt konstituieren (hier: Handlungsmöglichkeiten und -bedingungen auf der Wikipedia). Indem Macht unausweichlich mit 
individuellen Handlungen und übergeordneten Handlungsstrukturen verbunden ist, stellt sich hier die Frage, welche Handlungen bei der Generierung und Strukturierung von Wissensrepräsentationen auf der Wikipedia möglich sind und welchen Bedingungen diese unterliegen.

Bei der konkreten Analyse von Macht im Sinne Foucaults wird im Forschungsfeld wiederholt auf dessen Dispositivkonzept rekurriert. Er selbst hat dieses nur vage als »heterogene Gesamtheit, bestehend aus Diskursen, Institutionen, architektonischen Einrichtungen, reglementierenden Entscheidungen, Gesetzen, administrativen Maßnahmen, wissenschaftlichen Aussagen, philosophischen, moralischen und philanthropischen Lehrsätzen, kurz, Gesagtes ebenso wie Ungesagtes« (Foucault 2003, 392) definiert. In diesem Dispositivbegriff kommt das Netzwerk der Macht zum Ausdruck. Ebenso unterstellt Foucault ihm eine »dominant strategische Funktion« (ebd., 393). Deutlich wird dies an seinen Arbeiten zum Gefängnis- oder Sexualdispositiv. In beiden Fällen werden verschiedene Elemente wie Gefängnisstreik oder eine Transformation des Geschlechts als mögliche Handlungen in Abhängigkeit ihrer Bedingungen wie Richtlinien, Gesetzen oder architektonischen Einrichtungen gesetzt. Diese Verbindung bringt Gegenstände, materialisiert beim Gefängnis oder als Raum des Denkbaren gegenüber dem Geschlecht, erst hervor. Dispositive werden somit zum Analyseinstrument der produktiven Macht. Dieser Umstand hat sich bereits im sozialwissenschaftlichen Methodenarsenal niedergeschlagen (vgl. Jäger 2011; Bührmann und Schneider 2012) und findet sich selbst, wenn auch nur vereinzelt, in medienpädagogischen Diskussionen wieder (vgl. Bettinger und Dander 2016; Dander 2017).

Für die Transformation von Wissensaushandlung unter den Bedingungen der Digitalisierung ist anzumerken, dass sich etablierte Theorien und Konzepte zur Digitalisierung häufig auf gesellschaftliche Veränderungen beziehen. Zwar nehmen sie ihren Ausgangspunkt von digitalen Technologien wie Computer, Smartphone, Internet oder World Wide Web, verharren dabei jedoch nicht auf der technischen Perspektive. Inwieweit Technik unidirektional auf die Gesellschaft wirkt, bleibt in der vorliegenden Literatur oftmals implizit, wobei eine solche Tendenz unter anderem bei den Theorien zum Ende der GutenbergGalaxis (Bolz 1993), zum Postmodernen Wissen (Lyotard 2005 [1979]) oder zum Wikipedia-Universum (Pscheida 2010) auszumachen ist. Aus der Perspektive der produktiven Macht gilt es, eine solche Monokausalität zu vermeiden. 
Hierfür bietet sich das Dispositivkonzept an, welches sich über seinen Netzwerkcharakter von räumlich-technischen beziehungsweise technikzentrierten Konzepten unterscheidet. Digitalisiertes Wissen ist nicht ausschließlich über die räumlich-technische Anordnung von Produktion oder Rezeption erklärbar. Insbesondere vermögen Handlungsmöglichkeiten (im Kontext der Terminologie von Dispositivkonzepten auch: nicht-diskursive Praktiken), Handlungsbedingungen (auch: Objektivationen) und Handlungszuschreibungen (auch: diskursive Praktiken) zur Beschreibung der Wissensgenerierung und -strukturierung beitragen. Dabei kann die Anordnung von Websites und deren technische Bedingtheit durchaus von Bedeutung sein, jedoch nicht ausschließlich auf diese zurückgeführt werden.

Im Folgenden wird die produktive Macht der Wikipedia anhand von Handlungsmöglichkeiten und -bedingungen skizziert. Diese Machtanalytik umgeht somit die diskursiven Praktiken, also das »Gesagte«. Mit letzteren lassen sich unter anderem Zusammenhänge zwischen sprachlichem Handeln und gesellschaftlichen und institutionellen Strukturen herausarbeiten. Eine Analyse dieser Praktiken zielt dementsprechend auf kommunikativ hergestellte Sinnstrukturen. Diese Möglichkeit wird im Beitrag ausgespart, da der Fokus auf den medienspezifischen Kontextfaktoren in Form der Handlungsmöglichkeiten und -bedingungen der Wikipedia liegt.

Die folgende Beschreibung der produktiven Macht der Wikipedia beruht auf einer Dispositivanalyse (nach Bührmann und Schneider 2012) des Autors, welche im Frühjahr 2019 im Rahmen einer Dissertationsschrift durchgeführt wurde. ${ }^{1}$

Im Fall der Wikipedia entfaltet sich die produktive Macht in drei verschiedenen Typen der Wissensgenerierung, -prozessierung und -regulierung: 1) kollaborativ-meritokratisch, 2) expertokratisch-professionalisiert und 3) oligarchisch-technokratisch. Diese Typen vereinen dabei unterschiedliche Handlungsmöglichkeiten und -bedingungen. Sie ergänzen sich gegenseitig zu einem typisierenden Bild der Wissensgenerierung und -strukturierung der Wikipedia.

1 Eine überarbeitete Version der Dissertationsschrift erschien im Januar 2021 (Gemkow 2021). Die Dispositivanalyse teilt sich in die Analyse von diskursiven und nicht-diskursiven Praktiken sowie Objektivationen. Die diskursiven Praktiken wurden inhaltsanalytisch erhoben und ausgewertet. Bei den nicht-diskursiven Praktiken und den Objektivationen erfolgte eine Plattformanalyse auf der Wikipedia. 


\subsection{Kollaborativ-meritokratische Wissensformierung}

Der erste Typ, die kollaborativ-meritokratische Wissensformierung, zeichnet sich durch ein Zusammenspiel der beiden namensgebenden Elemente aus. Kollaborative Handlungsmöglichkeiten treten bei den verschiedenen Diskussions- und Regulierungsmechanismen hervor. Dabei wird deutlich, dass das Konzept in unterschiedliche Facetten zerfällt. Ihrer ursprünglichen Form nach bedeutet Kollaboration auf der Wikipedia, dass alle Nutzer*innen parallel und fortlaufend prinzipiell jeden Teil des potenziellen Endergebnisses (beispielsweise ein Artikel, ein Portal oder eine Kandidatur für einen bestimmten Nutzer*innenstatus) mitgestalten können. Tatsächlich handelt es sich dabei aber nur um einen Teilaspekt. In der Wikipedia werden Möglichkeiten zu dieser ursprünglichen Kollaboration durch hierarchische Elemente eingeschränkt. Benutzungsrechte regeln, wer zu bestimmten Arten der Mitgestaltung befugt ist und wer nicht. Hierdurch entsteht jedoch nichts anderes als eine Kollaboration auf höherer Ebene, für die es, je nach Zweck (etwa personelle Kandidatur, Gestaltung der Startseite oder Vandalismusbekämpfung) unterschiedliche Beteiligungsmöglichkeiten und -bedingungen gibt, da in jedem Fall der Grad der hierarchischen Elemente neu bestimmt wird.

Die beiden hierarchischen Elemente, die dabei zum Tragen kommen, sind zum einen die Zeit und zum anderen der geleistete Beitrag zur Wikipedia. Erstere ist über das Registrierungsdatum der jeweiligen Nutzer*innen bestimmt. Der geleistete Beitrag definiert sich hingegen durch Bearbeitungen und Kommentare auf den jeweiligen Seiten. Hierbei wird beispielsweise bei der Stimmberechtigung (vgl. Wikipedia 2020e) unterschieden, ob sich diese auf Artikel beziehen oder auf Diskussionsseiten.

Durch Einschränkung der parallelen und fortlaufenden Kollaboration auf mehreren Ebenen entsteht eine Form der Meritokratie, deren Prinzip zufolge die Leistungen des Individuums über dessen gesellschaftlichen Status innerhalb einer sozialen Gruppe bestimmen (vgl. Young 1961; Hoffer 2002; Hadjar 2008). Dennoch stellt die Kollaboration immer noch die vorherrschende Form der Wissensgenerierung und -prozessierung auf der Wikipedia dar. Sie wird jedoch durch hierarchische Elemente eingeschränkt, die sich nach dem Meritokratieprinzip legitimieren. Die hierarchischen Elemente sind zumindest im Zugang zu bestimmten Positionen obligatorisch, aber bei weitem nicht hinreichend, da Positionen auch durch Wahlen und damit wiederum durch Kollaborationen besetzt werden. 
Ein wichtiges Beispiel für eine kollaborativ-meritokratische Wissensformierung findet sich im Wettbewerb »Artikelmarathon«. Ziel dabei ist es, während eines festgelegten Zeitraums möglichst viele neue Artikel anzulegen. Die Nutzer*innen mit der höchsten Zahl gewinnen den Wettbewerb. Daran zeigt sich der meritokratische Fokus auf Leistung als zentralem Differenzierungskriterium, unabhängig von etwaigen anderen Legitimationsformen, wie Vorwissen oder Status. Der Wettbewerb beruht aber auch auf kollaborativen Elementen wie der Teilnahme anderer, den Artikelwunschlisten als Orientierung oder den (auch) kollaborativ erstellten Relevanzkriterien. Wie sich am Artikelmarathon ablesen lässt, ist Meritokratie auf der Wikipedia nur im Zusammenspiel mit Kollaboration zu verstehen.

Auffallend ist auch, dass meritokratische Elemente dort eher in quantitativer als in qualitativer Form umgesetzt werden. Bei beiden hierarchischen Elementen, Zeitraum und Beiträgen, stehen die bloße Dauer beziehungsweise Anzahl im Mittelpunkt. Da die Wikipedia aber auch inhaltliche Qualitätsbausteine hat (wie Wettbewerbe oder Kandidaturen), wäre ein zusätzliches meritokratisch qualitatives Element denkbar.

\subsection{Expertokratisch-professionalisierte Wissensformierung}

Der zweite produktive Machttypus, die expertokratisch-professionalisierte Wissensformierung, zeichnet sich durch das Zusammenspiel von expertokratischen Elementen mit und in Formen der Professionalisierung aus. In der Wikipedia sind Formen der Vergemeinschaftung zu verzeichnen, die sich thematisch deutlich spezifizieren. Dabei handelt es sich um eine Form der Kollaboration, bei der aus der Gruppe heraus Wissen generiert und prozessiert wird. Da die Wikipedia zum jetzigen Zeitpunkt schon sehr ausdifferenziert ist, werden in den Vergemeinschaftungen spezifische Themen verhandelt: Ein Portal über die Sezessionskriege gehört ebenso dazu wie eines über den Kampfsport Budō. Dadurch entsteht das Bild von vergemeinschafteten Expert*innen. Die Expertokratie wird auch über andere Elemente befördert, wie beispielsweise durch feste Ansprechpartner*innen für bestimmte Themen, den Einsatz von Tutorien als Form der Einweisung in die Wikipedia oder auch die Funktion »Artikel on Demand «, mit der sich Autor*innen selbst als Expert*innen für ein Thema ausweisen können. Spezifische Ansprechpartner*innen oder Tutor*innen fungieren in ihrer Rolle als 
Schnittstelle zwischen einem konkreten Thema oder einer Funktion der Wikipedia und den jeweiligen Nutzer*innen, die fragend an sie herantreten. Dadurch werden sie zu Expert*innen für Themen an sich und die Wikipedia selbst. Weiterhin können Nutzer*innen durch die sogenannten »Babel-Bausteine « ihre Fähigkeiten, thematisches Wissen, Qualifikationen und Leistungen sowie Funktionen für andere sichtbar machen. Mittels dieser potenziellen Selbstreferenz besteht abermals die Möglichkeit, expertokratische Strukturen in die Wikipedia einzubringen.

Diese werden insbesondere durch die inhaltliche Segmentierung der Plattform befördert. Ähnlich einem klassischen Fächerkanon ist die Wikipedia in verschiedene Portale und Themenfelder gegliedert. Eine solche kanonisierte und ausdifferenzierte Strukturierung von Wissen macht Formen der Reglementierung unabdingbar, die in den bereits erwähnten Vergemeinschaftungen stattfindet. Diese verhandeln die Generierung von neuem Wissen, indem sie Artikelwünsche aufbauen und verwalten. Ebenso verhandeln sie die Prozessierung von bestehendem Wissen, indem sie auf qualitative und quantitative Mängel an bestehenden Artikeln hinweisen. Durch die thematische Spezifität werden hier expertokratische Strukturen sichtbar. Daneben verweisen unter anderem diese Vergemeinschaftungen auch auf einen einsetzenden Professionalisierungsprozess auf der Wikipedia.

Unter letzterem wird eine Verberuflichung verstanden, wobei die Profession auf bestimmte erreichte Standards verweist (vgl. Evetts 2003). Auch wenn diese Maßstäbe unterschiedlich ausgelegt werden (beispielsweise Qualifikationsmöglichkeiten, Interessenvertretung oder gesetzlicher Rahmung), zeigen sich auf der Wikipedia klare Professionalisierungsmuster. So ist es etwa durch den sukzessiven Aufstieg innerhalb der hierarchisch-meritokratischen Benutzungshierarchie möglich, eine - zumindest intern anerkannte - »Karriere« zu durchlaufen. Weiterhin verfügt die Wikipedia über Richtlinien und Konventionen, die als Grundlage der Wissensgenerierung und -strukturierung für alle leitend und einsehbar sind. Individuelle Förderung ist durch die verschiedenen Hilfestellungen in Form von Tutor*innen, Mentor*innen und Ansprechpartner*innen ebenfalls gegeben. Für eine etablierte Interessensvertretung spricht die Implementation von Schlichtern und eines Schiedsgerichts im Streitfall. 


\subsection{Oligarchisch-technokratische Wissensformierung}

Der dritte produktive Machttypus auf der Wikipedia ist der oligarchisch-technokratische. Als Oligarchie wird in der Politikwissenschaft eine Herrschaftsform bezeichnet, »in der eine kleine Gruppe von wenigen Personen auf Dauer Macht ausübt« (Herzog 2004, 609). Eine solche tritt auf der Wikipedia in zwei unterschiedlichen Formen auf.

Die erste vereint die beiden bereits beschriebenen Typen, kollaborativ-meritokratisch und expertokratisch-professionalisiert, und verdeutlicht, dass sich hierarchische Elemente durch das Meritokratieprinzip verhärten. Dies geschieht außerdem über spezialisierte Vergemeinschaftungen, in denen sich professionalisierte Formen der Aufgabenteilung und Rollenverteilung entwickeln sowie Gruppen von wenigen Nutzer*innen entstehen, die ein höheres Maß an Benutzungsrechte besitzen. In der Wikipedia trifft das unter anderem auf die Gruppe der Administrator*innen zu, die beispielsweise über erweiterte Funktionen wie das Löschen und Wiederherstellen von Dateien und Versionen, das Sperren von Benutzungskonten und Diskussionsseiten oder die Bearbeitung von Spezialseiten verfügen. Ihr gehören in Deutschland 182 Nutzer*innen (Stand: 20.06.2020; vgl. Wikipedia 2020c) an. Bei knapp über drei Millionen angemeldeten Mitgliedern ergibt das einen Anteil von 0,006 Prozent oder anders formuliert: Auf eine*n Administrator*in kommen rund 17530 registrierte Mitglieder. Diese Zahlen verdeutlichen das ungleiche Verhältnis zwischen Nutzer*innen mit erweiterten Rechten und Nutzer*innen ohne. Da erstere mehr Entscheidungsgewalt haben (wie etwa bei Wahlen, der Implementierung von neuen Funktionen, Sperrungen von Versionen und Konten) und auch Absprachen nicht auszuschließen sind, kann hier von einem oligarchischen Machttypus gesprochen werden.

Die zweite oligarchische Form auf der Wikipedia wird anhand der Einflussnahme der Wikimedia Foundation deutlich. Sie ist eine gemeinnützige Organisation im Sinne des US-amerikanischen Gesetzes und hält alle Rechte an den verschiedenen nationalsprachlichen Versionen sowie deren Namen, Domains und Servern. In dieser Position nehmen sie und ihre Mitarbeiter*innen in unterschiedlicher Weise Einfluss auf die deutschsprachige Wikipedia und das dort repräsentierte Wissen. Die Wikimedia Foundation hat in ihren »unumstößliche(n)« Grundprinzipien (Wikipedia 2020b) Richtlinien für das Selbstverständnis der Wikipedia festgelegt, auf denen aufbauend sich alle Handlungsmöglichkeiten und -bedingungen entwickelt haben. Weiterhin 
bestimmt sie, wofür ein Benutzungskonto notwendig ist, und stützt die hierarchischen Strukturen über verschiedene Richtlinien, die für Benutzungsgruppen mit erweiterten Rechten bindend sind (beispielsweise die Oversight- oder Stewards-Policy).

Auch die Technik obliegt der Wikimedia Foundation und dem Einfluss von Benutzungsgruppen mit erweiterten Rechten. Alle Features der Wikipedia werden entweder von freischaffenden Developern entwickelt und von der Organisation eingebunden oder direkt von internen Mitarbeiter*innen erarbeitet. Neben der Software und der Benutzungsoberfläche ist auch die Hardware in Form von Servern, dem technischen Grundstein für die Kommunikation und Archivierung der gesamten Wikipedia, in der Hand der Wikimedia Foundation.

Ihre Rolle kommt somit einer oligarchischen Arbeitsorganisation gleich. Die Wikimedia Foundation nimmt über ihre Grundprinzipien, Richtlinien und ihre Technikbestimmtheit indirekt Einfluss auf das von der Wikipedia repräsentierte Wissen. Ebenso sind die hierarchischen Benutzerstrukturen Pfeiler eines oligarchisch anmutenden Systems, welches mit der technokratischen Hervorbringung und Prozessierung von Wissen in Form und mit Hilfe von Bots, Features und der Legitimierung von Wissen durch Expertise produktive Macht entfaltet.

\section{$4 \quad$ Zur Rolle der Medienkompetenz}

Durch kommunikative Medientechnologien werden sowohl neue Möglichkeiten als auch Bedingungen für Medienhandeln bereitgestellt. Innerhalb der Wikipedia entsteht somit eine produktive Macht, die nicht nur mediale Wissensrepräsentationen hervorbringt, sondern über die spezifische Art der Hervorbringungen auch die Rolle von Akteur*innen, Institutionen, Richtlinien und Techniken zueinander in Beziehung setzt. Für die Medienpädagogik stellt sich hier die Frage, ob das Konzept der Medienkompetenz diese spezifische Hervorbringungsweise adressieren kann. Dafür ist ein Blick auf sein theoretisches Fundament vonnöten.

Der Linguist Noam Chomsky hat den Kompetenzbegriff ursprünglich mit dem Spracherwerb verbunden. Kompetent zu sein besteht für ihn in nichts anderem als der Fähigkeit, gesprochene Sprache erlernen zu können. Sprache ist für Chomsky ein Zeichensystem, welches hierarchisch und regelgeleitet geordnet ist. Die allen Sprachen zugrundeliegende Regelhaftigkeit wird als Universalgrammatik bezeichnet. Dabei 
liegt die Kompetenz des Menschen darin, allein durch seine Gattungszugehörigkeit die Grammatik einer gegebenen natürlichen Sprache erlernen zu können. Nur so sei es erklärbar, dass Kinder Sätze bilden können, die sie noch nie zuvor gehört haben. Chomskys Kompetenzbegriff ist nicht auf die tatsächliche Verwendung hin ausgerichtet, sondern stellt ein theoretisches Modell für eine unbewusste anthropologische Fähigkeit dar. Kompetenz wird mithin zu einem idealen und subjektzentrierten Konzept.

Habermas (1971) bezieht sich in der Theorie des kommunikativen Handelns auf diesen Kompetenzbegriff, rückt ihn jedoch von einem biologischen zu einem soziologischen Verständnis. Bei ihm sind Kompetenzen nicht mehr nur von Natur aus gegeben, sondern auch sozial strukturiert und entwickeln sich in intersubjektiver Verständigung. Diese analysiert er mit Hilfe der Sprechakttheorie. In Sprechakten könnten Subjekte Geltungsansprüche wie Wahrheit, Richtigkeit oder Wahrhaftigkeit bezüglich objektiver, sozialer oder subjektiver Gegenstände erheben und anzweifeln. Habermas unterstellt der menschlichen Interaktion jedoch eine ideale Sprechsituation (vgl. Habermas und Luhmann 1971, 136). Der Zweifel an den geäußerten Geltungsansprüchen von Sprecher*innen sei zugleich ein Zweifel an deren kommunikativer Kompetenz. Nach Habermas müsste man nun dem Zweifel in Form einer diskursiven Prüfung genauer nachgehen. Der Diskurs selbst sei jedoch ebenfalls ein Sprechakt, der wiederum angezweifelt werden könne. Dafür bedürfe es eines übergeordneten Diskurses, welcher die Geltungsansprüche des ursprünglichen prüft. Aber ein solcher Vorgang mündet in einem infiniten Regress der Prüfung. Dieser ist laut Habermas nicht nötig, da eine ideale Sprechsituation in jeder Kommunikation zwischen Menschen unterstellt wird (vgl. ebd.). Somit bleibt auch bei ihm der idealistisch-subjektzentrierte Impetus von Kompetenz bestehen.

Dieter Baacke sieht darauf aufbauend die Notwendigkeit zur kommunikativen Kompetenz. Gemäß Baacke $(1975,363)$ sei der Mensch ein »Homo educandus «, also ein erziehungsfähiges, aber auch -bedürftiges Lebewesen. Die Ausprägung der kommunikativen Kompetenz bleibt »ein Spielraum des Menschen für die Bildung unendlich vieler Sätze wie für potenziell unbegrenztes Kommunikationsverhalten« (ebd., 101-102). In diesem Sinne tritt sie wiederum als ein idealistischsubjektzentriertes Konzept auf, da sie dem Mensch Kompetenz unterstellt und diese somit zu einer grundlegenden anthropologischen Eigenschaft macht (vgl. Baacke 1994, 240). 
In Baackes Sinn soll Medienkompetenz hier als Teil der kommunikativen Kompetenz verstanden werden. Er definiert sie als »Fähigkeit, in die Welt aktiv aneignender Weise auch alle Arten von Medien für das Kommunikations- und Handlungsrepertoire von Menschen einzusetzen« (Baacke 1996a, 8). Mit seiner Berücksichtigung von Medien reagiert Baacke auf »die Veränderung der Kommunikationsstrukturen durch technisch industrielle Vorkehrungen und Erweiterungen« (Baacke 1996b, 119). Damit akzentuiert Medienkompetenz als Teil der kommunikativen Kompetenz den Aspekt der Medienkommunikation, der gerade "nicht in face-to-face Situationen stattfindet, sondern in parasozialen Interaktionen mit Medienbotschaften und ihren Trägern« (Baacke 1997, 54).

Chomsky hatte mit der generativen Grammatik eine biologische Determinante geschaffen, die grundsätzlich universell und idealistischsubjektzentriert ausgelegt ist. Diese Attribute ziehen sich durch die kommunikative Kompetenz bei Habermas bis zum Konzept der Medienkompetenz bei Baacke und dessen medienpädagogischem Verständnis (vgl. Gapski 2001, Kerres 2011, Bettinger und Aßmann 2017).

Die produktive Macht in der Wikipedia zeigt ein spezifisches Kräfteverhältnis verschiedener Akteur*innen. Dieses ist spezifisch für jede Plattform beziehungsweise jedes mediale Angebot und bestimmt Handlungsmöglichkeiten und -bedingungen. Medienkompetenz muss daher die jeweilige Medienspezifik reflektieren. Es gilt, sich mit den jeweils gegebenen Medienumgebungen spezifisch auseinanderzusetzen, da Handlungsmöglichkeiten erst in diesen auf ihre Struktur hin untersucht werden können. Dies schließt für das Konzept der Medienkompetenz aber auch ein, die produktive Macht von Plattformen wie der Wikipedia empirisch zu untersuchen, um ihnen gegebenenfalls ein adäquates medienpädagogisches Korrektiv gegenüberzustellen.

Wenn die Wikipedia Wissen über kollaborativ-meritokratische Machtstrukturen repräsentiert, dann gilt es, diese entsprechend zu adressieren. Das bedeutet allgemein erst einmal zu erkennen, dass dort eine Verknüpfung zwischen Kollaboration und Meritokratie besteht. Diese zeigt sich in unterschiedlichen Handlungsmöglichkeiten und -bedingungen wie einem Kollaborationszwang, der über Wunschlisten, Portale und Wettbewerbe aufrechterhalten wird. Ebenso gilt es zu erkennen, dass hierarchisch strukturierte Vergemeinschaftungen bestehen, wobei sich die Hierarchie, wie oben ausgeführt, auf meritokratische Aspekte stützt. Medienkompetenz muss sich dann aber auch auf das Medienhandeln stützen. Dafür können ebenfalls Kompetenzen 
konkretisiert werden, wie beispielsweise das Anbieten eigener Qualitäten über Kandidaturenteilnahme, Software-Entwicklung, Teilnahme am Mentorenprogramm, Hilfestellung für Neulinge oder die Erstellung neuer Vergemeinschaftungen bis hin zur Erhöhung des Mitspracherechts bei bestehenden Kontrollinstanzen.

Für die medienpädagogische Forschung bedeutet dies, dass die Modellierung und Operationalisierung von Medienkompetenz auf medienspezifische Aspekte angewiesen ist. Das heißt, dass Medienkompetenzmodelle entwickelt werden müssen, die sich an medienspezifischen sowie auch thematischen Besonderheiten orientieren.

\section{Das Transformieren der Transformation}

Die Debatten um die Folgen der Digitalisierung von Wissen für dessen Generierung und Prozessierung sind ganz unterschiedlich datiert. Während Lyotard beispielsweise bereits 1979 mit Blick auf die Verbreitung des Computers von einer »technologischen Transformation [...] auf das Wissen « (Lyotard 2005 [1979], 21) sprach, sind bis heute andere Marksteine wie das Web 2.0 (Pscheida 2010) oder die Wiki-Technologie (Iske und Marotzki 2010) hinzugekommen. Sofern der Begriff »Transformation« im Zusammenhang mit medial repräsentiertem Wissen einen Umbruch von einer historisch und soziotechnisch spezifischen Konstellation zu einer anderen bedeutet, dann kann mit Blick auf die weitreichenden Diskussionen um die Digitalisierung von Wissen nicht von einer abgeschlossenen Transformation gesprochen werden. Eine solche abgeschlossene Transformation - wie sie mit den Theorien der Informations-, Wissens- oder Netzwerkgesellschaft assoziiert wird ist für die Dynamik, mit der sich Plattformen wie die Wikipedia verändern können, schwer denkbar. Dementsprechend scheint es konsequent, im Fall der Digitalisierung die Perspektive der Mediatisierung aufzugreifen und von einem Transformationsprozess zu sprechen. Das bedeutet, dass es sich bei letzterem gerade nicht um eine »räumlich und zeitlich umgrenzte Entwicklung mit einem klaren Anfangs- und Endpunkt« (Krotz 2007, 11) handelt. Neben der räumlichen und zeitlichen Entgrenzung gibt es auch keine Begrenzung von sozialen und kulturellen Folgen (vgl. ebd., 12). Diese Langzeitentwicklung ist zwar abhängig von kulturellen Kontexten, aber weder monokausal noch linear erklärbar (vgl. Krotz 2017, 27). Mediatisierung wird in dieser Lesart dezidiert von zeitlich und räumlich begrenzten Ansätzen unterschieden, die ver- 
suchen, gesellschaftliche Entwicklung monokausal zu erklären. Dies öffnet den Blick auf die Digitalisierung als transformativen Mediatisierungsschub (vgl. Hepp und Pfadenhauer 2014, 236).

Gegenwärtig treten eine Vielzahl von sich gegenseitig beeinflussenden Transformationsprozessen auf. Zu nennen sind neben der Digitalisierung (Zink 2019) die Flexibilisierung (Streeck 2011), Ökonomisierung (Beyer 2009) oder Subjektivierung (Moldaschl und Voss 2003). Erstere muss demnach als ein umfassender gesellschaftlicher Transformationsprozess verstanden werden. Eine auf Einzelmedien sowie individuelle Nutzer*innen begrenzte Perspektive greift ebenso zu kurz wie eine rein technologische. In diesem Sinne sind auch die in diesem Beitrag skizzierten Ergebnisse zur produktiven Macht der Wikipedia nur eine Momentaufnahme. Diese versucht jedoch, Transformation in einer dynamischen Perspektive mit mehreren Akteur*innen und unterschiedlichen Handlungsbedingungen zu fassen. Für das Konzept der Medienkompetenz bedeutet diese theoretische Auslegung eine Anpassungsleistung - weg von idealistisch-subjektzentrierten Normen hin zu dynamischen, medienspezifischen Kompetenzaspekten.

\section{$6 \quad$ Fazit}

Mit dem Konzept der produktiven Macht geht der Beitrag einen Mittelweg zwischen dem Fokus auf medienspezifischen Handlungsmöglichkeiten einerseits und medienspezifischen Handlungsbedingungen andererseits. Statt, wie beispielsweise für sozialkonstruktivistische kommunikationswissenschaftliche Forschung typisch, allein nach konkreten aneignenden Interaktionszusammenhängen $\mathrm{zu}$ fragen, stellt der Ansatz dieses Beitrages die Bedingungen für Kommunikation in den Vordergrund. Diese ermöglichen nicht nur die Aneignung selbst, sondern strukturieren auch ihren Gegenstand. Die medienspezifischen Handlungsmöglichkeiten und -bedingungen sind die Wissensformierungen. Analog zu anderen Arbeiten der jüngeren Kommunikationswissenschaft, die beispielsweise das Dispositivkonzept (vgl. Lepa et al. 2014), die Akteur-Netzwerk-Theorie (vgl. Gentzel 2013) oder die materialistische Phänomenologie (vgl. Hepp 2018) anwenden, vertritt dieser Beitrag den Ansatz, dass eine Trennung von autark handelndem Subjekt auf der einen und handlungsdeterminierenden Medien(-technologien) auf der anderen Seite dem Wandel der Digitalisierung nicht gerecht wird. Mit dem Zugang über die Wikipe- 
dia entsteht somit eine spezifische Beschreibung der Wissensaushandlung, die sich gerade durch ihre soziotechnische Konstellation als transformativ zu früheren oder alternativen Formen der Wissensaushandlung verstehen lässt.

Für die Medienkompetenz im Speziellen hat diese theoretische Entwicklung selbst große Bedeutung. Schließlich geht es hier um die Frage, welchen Raum medientechnische Entwicklungen einnehmen und wie das Subjekt dabei gedacht werden kann. Die Stellung des idealisierten Subjekts wurde in der Medienpädagogik in den letzten Jahren immer wieder kritisch reflektiert (vgl. Dander 2017; Kammerl 2017). Die tiefgreifende Durchdringung des Alltags mit digitalen Medien - hier beispielsweise die Wissensaneignung - gestattet jedoch nicht mehr, eine Trennung von Mensch und Medium anzunehmen. Handlungsmöglichkeiten und -bedingungen mit digitalen Wissensrepräsentationen sind nicht medien- oder subjektzentriert zu denken. Sie entwickeln sich vielmehr im heterogenen Spiel verschiedener Akteur*innen zueinander und sind dabei stets wandelbar. Das medienkompetente Subjekt muss sich demnach auch immer kritisch zu seinem eigenen mediatisierten Handeln in Beziehung setzen können. Inwieweit es sich dann als autonom, selbstbestimmt und aufgeklärt begreift, wird mithin selbst zum Gegenstand der eigenen Medienkompetenz.

\section{Literatur}

Arnold, Patricia, Lars Kilian, Anne Thillosen, und Gerhard Zimmer. 2018. Handbuch E-Learning: Lehren und Lernen mit digitalen Medien. 5. Auflage. Bielefeld: Bertelsmann.

Baacke, Dieter. 1975. Kommunikation und Kompetenz: Grundlegung einer Didaktik der Kommunikation und ihrer Medien. 2. Auflage. München: Juventa.

Baacke, Dieter. 1996a. Medienkompetenz als Netzwerk: Reichweite und Fokussierung eines Begriffs, der Konjunktur hat. medien praktisch 20 (78): 4-10.

Baacke, Dieter. 1996b. Medienkompetenz: Begrifflichkeit und sozialer Wandel. In: Medienkompetenz als Schlüsselbegriff, herausgegeben von Antje von Rein, 112124. Bad Heilbrunn: Klinkhardt.

Baacke, Dieter. 1997. Medienpädagogik. Grundlagen der Medienpädagogik. Tübingen: Niemeyer.

Baacke, Dieter. 1998. Zum Konzept der Operationalisierung von Medienkompetenz. http://www.produktive-medienarbeit.de/ressourcen/bibliothek/fachartikel/baacke_operationalisierung.shtml. Zugegriffen: 20. Juni 2020.

Bettinger, Patrick, und Sandra Aßmann. 2017. Das diskursive Feld um Mediatisierung und Mediensozialisation: Eine Analyse deutschsprachiger Fachzeitschrif- 
ten. In: Mediatisierung und Mediensozialisation: Prozesse - Räume - Praktiken, herausgegeben von Dagmar Hoffmann, Friedrich Krotz und Wolfgang Reißmann, 79-100. Wiesbaden: Springer VS.

Bettinger, Patrick, und Valentin Dander. 2016. Das Dispositiv als Bindeglied einer interdisziplinären Medienbildungsforschung. Rezension. MedienPädagogik: Zeitschrift für Theorie und Praxis der Medienbildung. DOI: https://doi. org/10.21240/mpaed/XX/2016.09.12.X.Dander 2017.

Beyer, Jürgen. 2009. Varietät verspielt? Zur Nivellierung der nationalen Differenzen des Kapitalismus durch globale Finanzmärkte. In: Wirtschaftssoziologie. Sonderheft der Kölner Zeitschrift für Soziologie und Sozialpsychologie 49, herausgegeben von Jens Beckert und Christoph Deutschmann, 305-325. Wiesbaden.

Bolz, Norbert. 1995. Am Ende der Gutenberg-Galaxis. München: Fink.

Bührmann, Andrea, und Werner Schneider. 2012. Vom Diskurs zum Dispositiv: Eine Einführung in die Dispositivanalyse. Bielefeld: Transcript.

Chomsky, Noam. 1970. Aspekte der Syntax-Theorie. Berlin: Akademie-Verlag.

Cunningham, Ward. 2002. What is a Wiki. http://www.wiki.org/wiki.cgi?WhatIsWiki. Zugegriffen: 16. August 2020.

Dander, Valentin. 2017. Medien - Diskurs - Kritik: Potenziale der Diskursforschung für die Medienpädagogik. In: Spannungsfelder und blinde Flecken: Medienpädagogik zwischen Emanzipationsanspruch und Diskursvermeidung, herausgegeben von Sven Kommer, Thorsten Junge und Christiane Rust, 50-61.

Foucault, Michel. 1978. Dispositive der Macht: Über Sexualität, Wissen und Wahrheit. Berlin: Merve.

Foucault, Michel. 1994. Das Subjekt und die Macht. In: Michel Foucault: Jenseits von Strukturalismus und Hermeneutik, herausgegeben von Hubert L. Dreyfus und Paul Rabinow, 2. Auflage, 243-261. Weinheim: Beltz-Athenäum.

Foucault, Michel. 1996. Der Mensch ist ein Erfahrungstier: Gespräch mit Ducio Trombadori. Frankfurt am Main: Suhrkamp.

Foucault, Michel. 2002. Schriften. In: vier Bänden = Dits et écrits. Band 2, herausgegeben von Daniel Defert und François Ewald, 2. Auflage. Frankfurt am Main: Suhrkamp.

Foucault, Michel. 2003. Das Spiel des Michel Foucault. In: Schriften. In: vier Bänden = Dits et écrits, herausgegeben von Daniel Defert und Michael Bischoff, 391429. Frankfurt am Main: Suhrkamp.

Foucault, Michel. 2005. Schriften. In: vier Bänden = Dits et écrits. Band 4, herausgegeben von Daniel Defert und François Ewald, 2. Auflage. Frankfurt am Main: Suhrkamp.

Gapski, Harald. 2001. Medienkompetenz: Eine Bestandsaufnahme und Vorüberlegungen zu einem systemtheoretischen Rahmenkonzept. Wiesbaden: Westdeutscher Verlag.

Gemkow, Johannes. 2017: Medienkompetenz und die Mediatisierung des Wissens: Zum Potenzial der Dispositivanalyse am Beispiel mediatisierter Wissensbestände. merzWissenschaft 61 (6): 18-29.

Gemkow, Johannes. 2021. Die Mediatisierung des Wissens: Eine Dispositivanalyse zur Rolle der Medienkompetenz. Wiesbaden: Springer VS. 
Gentzel, Peter. 2013. Praxistheorie und Mediatisierung: Grundlagen, Perspektiven und eine Kulturgeschichte der Mobilkommunikation. Wiesbaden: Springer VS.

Habermas, Jürgen, und Niklas Luhmann. 1971. Theorie der Gesellschaft oder Sozialtechnologie. Frankfurt am Main: Suhrkamp.

Hadjar, Andreas. 2008. Meritokratie als Legitimationsprinzip: Die Entwicklung der Akzeptanz sozialer Ungleichheit im Zuge der Bildungsexpansion. Wiesbaden: Springer VS.

Hepp, Andreas. 2018. Von der Mediatisierung zur tiefgreifenden Mediatisierung. Konstruktivistische Grundlagen und Weiterentwicklungen in der Mediatisierungsforschung. In: Kommunikation - Medien - Konstruktion: Braucht die Mediatisierungsforschung den Kommunikativen Konstruktivismus?, herausgegeben von Jo Reichertz und Richard Bettmann, 27-46. Wiesbaden: Springer VS.

Hepp, Andreas, und Michaela Pfadenhauer. 2014. Mediatisierte Partizipation? Kleine Formen der Beteiligung jenseits von Medienlogik. In: Die Mediatisierung sozialer Welten: Synergien empirischer Forschung, herausgegeben von Friedrich Krotz, Cathrin Despotović und Merle-Marie Kruse, 235-262. Wiesbaden: Springer VS.

Herzog, Dietrich. 2004. Oligarchie. In: Lexikon der Politikwissenschaft, herausgegeben von Dieter Nohlen und Rainer-Olaf Schultze, 609. München: Beck.

Hoffer, Thomas. 2002. Meritocracy. In: Education and sociology: An encyclopedia, herausgegeben von David Levinson, Peter Cookson und Alan Sadovnik, 435442. New York: RoutledgeFalmer.

Iske, Stefan, und Winfried Marotzki. 2010. Wikis: Reflexivität, Prozessualität und Partizipation. In: Medienbildung in neuen Kulturräumen: Die deutschsprachige und britische Diskussion, herausgegeben von Ben Bachmair, 141-151. Wiesbaden: VS Verlag.

Kammerl, Rudolf. 2017. Das Potential der Medien für die Bildung des Subjekts: Überlegungen zur Kritik der Subjektorientierung in der medienpädagogischen Theoriebildung. MedienPädagogik: Zeitschrift für Theorie und Praxis der Medienbildung. DOI: https://doi.org/10.21240/mpaed/27/2017.01.14.X.

Kerres, Michale. 2011. Schule und Internet: Pädagogische Aspekte der Entwicklung von Kompetenz für aktives Medienhandeln. RdJB - Recht der Jugend und des Bildungswesens 58 (4): 416-435.

Krotz, Friedrich. 2007. Mediatisierung: Fallstudien zum Wandel von Kommunikation. Wiesbaden: VS Verlag.

Krotz, Friedrich. 2017. Sozialisation in mediatisierten Welten: Mediensozialisation in der Perspektive des Mediatisierungsansatzes. In: Mediatisierung und Mediensozialisation: Prozesse - Räume - Praktiken, herausgegeben von Dagmar Hoffmann, Friedrich Krotz und Wolfgang Reißmann, 21-40. Wiesbaden: Springer VS.

Lepa, Steffen, Friedrich Krotz, und Anne-Kathrin Hoklas. 2014. Vom »Medium« zum »Mediumdispositiv«: Metatheoretische Überlegungen zur Integration von Situations- und Diskursperspektive bei der empirischen Analyse mediatisierter sozialer Welten. In: Die Mediatisierung sozialer Welten: Synergien empirischer Forschung, herausgegeben von Friedrich Krotz, Cathrin Despotović und MerleMarie Kruse, 115-141. Wiesbaden: Springer VS. 
Lyotard, Jean-François. 2005 [1979]. Das postmoderne Wissen: Ein Bericht. Unter Mitarbeit von Peter Engelmann. 5. Auflage. Wien: Passagen-Verlag.

Moldaschl, Manfred, und G. Günter Voss. 2003. Subjektivierung von Arbeit. 2. Auflage. München: Hampp.

Pscheida, Daniela. 2010. Das Wikipedia-Universum: Wie das Internet unsere Wissenskultur verändert. Berlin: De Gruyter.

Schiefner-Rohs, Mandy. 2012. Kritische Informations- und Medienkompetenz: Theoretisch-konzeptionelle Herleitung und empirische Betrachtungen am Beispiel der Lehrerausbildung. Münster: Waxmann.

Streeck, Wolfgang. 2011. Flexible employment, flexible families, and the socialization of reproduction. In: Imploding populations in Japan and Germany: A comparison, herausgegeben von Florian Coulmas und Ralph Lützeler, 63-95. Leiden, Boston.

Walden, Thomas. 2016. Medienkompetenz 2.1. In: Ent-Grenztes Heranwachsen, herausgegeben von Ulrike Becker, Henrike Friedrichs, Friederike von Gross und Sabine Kaiser, 233-251. Wiesbaden: Springer VS.

Wikipedia. 2020a. Größenvergleich. https://de.wikipedia.org/wiki/Wikipedia:Wikipedistik/Wachstumsprognose. Zugegriffen: 20. Juni 2020.

Wikipedia. 2020b. Ignoriere alle Regeln. https://de.wikipedia.org/wiki/Wikipedia:Ignoriere_alle_Regeln. Zugegriffen: 20. Juni 2020.

Wikipedia. 2020c. Liste der Administratoren. https://de.wikipedia.org/wiki/Wikipedia:Liste_der_Administratoren. Zugegriffen: 20. Juni 2020.

Wikipedia. 2020d. Statistics. http://wikistats.wmflabs.org/display.php?t=wp. Zugegriffen: 20. Juni 2020.

Wikipedia. 2020e. Stimmberechtigung. https://de.wikipedia.org/wiki/Wikipedia:Stimmberechtigung. Zugegriffen: 20. Juni 2020.

Young, Michael. 1961. Es lebe die Ungleichheit: Auf dem Wege zur Meritokratie. Düsseldorf: Econ-Verlag.

Zink, Klaus J. 2019. Arbeit und Organisation im digitalen Wandel. Baden-Baden.

Zorn, Isabel. 2011. Medienkompetenz und Medienbildung mit Fokus auf Digitale Medien. In: Medienbildung und Medienkompetenz: Beiträge zu Schlüsselbegriffen der Medienpädagogik, herausgegeben von Heinz Moser, Petra Grell und Horst Niesyto, 175-209. München: kopaed.

\section{Open Access}

Dieser Beitrag erscheint unter der Creative-Commons-Lizenz CC BY-ND 3.0 DE: https://creativecommons.org/licenses/by-nd/3.0/de/. 